\title{
Caractérisation des co-produits du raffinage valorisation énergétique ${ }^{*}$
}

\author{
Marc LAMBERT \\ De Smet Ballestra, \\ c/o M. Lambert, resp. environnement, \\ Da Vincilaan 2, boite G1, \\ B-1935 ZaventemZaventem, Belgique \\ Fax : +32 27161109 \\ $<$ ml@desmetgroup.com>
}

\begin{abstract}
A cost effective alternative to the existing treatment steps of the refining by-ptoducts, is the "on site" energetical valorization through 2 comparable technologies: thermal oxidation and anaerobic digestion combined with boiler. In a thermal multifluid oxidizer Cinclus ${ }^{\circledR}$, the mixture is injected with atomisation and combustion air at high velocity into the oxidation chamber. The jet creates a local under-pressure and thus an internal recirculation in the oxidation chamber. This results in a controlled and homogeneous temperature and residence profile that ensure an optimal combustion with low emissions. To prevent persistent boiler fouling, the gases are cooled to below $750{ }^{\circ} \mathrm{C}$ in the gas conditioning chamber by mixing the hot gases with recycled gases taken from behind the de-dusting device. Divert investment for fossil fuel boiler and soap stock splitting to install Cinclus soap stock boiler will allow: no acid waste water, no interphase, no sulfuric acid consumption, no fossil fuel consumption. The Dranco process is a well-known and reliable anaerobic digestion technology. Its simple digester design, no moving parts within the digester, provides a high reliability and low maintenance costs. The Dranco process parameters are roughly: digester loading: 10 to $20 \mathrm{~kg} C O D / \mathrm{m}^{3}$ reactor.day. The temperature range of the thermophilic organisms is 50 to $58{ }^{\circ} \mathrm{C}$. The retention time in the digester is about 15 to 30 days. The biogas production is above $300 \mathrm{~m}^{3}$ of biogas per ton of waste. The electricity production: above $500 \mathrm{kWh}$ per ton of waste. The advantages of anaerobic digestion of waste are: The production of renewable energy and reduction of fossil $\mathrm{CO}_{2}$-emissions; the low odour emissions and in general low emissions towards the environment.
\end{abstract}

Key words: energetical valorization, refining by-products, thermal oxidation, anaerobic digestion

Ballestra, de manière à propos leurs clients des usines écologiques et durables, étudie la faisabilité de traiter les co-produits issus du raffinage sur le site industriel-même.

Par « co-produits » (cop) sont visés :

Dans la catégorie non toxiques:

- les gommes (acides et lécithine) ;

- les pâtes de neutralisation ;

- les terres décolorantes (décoloration).

Et considérés comme toxiques:

- les adsorbants : charbon actif ;

- les distillats d'acides gras (FAD) (désodorisation);

- les terres décolorantes Nickel (hydrogénation).

L'objectif est de récupérer le potentiel énergétique énorme contenu dans les cop du raffinage au moyen de deux techniques:

\footnotetext{
* Texte de la conférence prononcée dans le cadre de la Journée de l'AFECG, sur les « Ingrédients bioactifs issus des huiles végétales et marines » du 10 octobre 2005, Paris.
}

Tableau 1. Volumes européens de co-produits du raffinage. l'oxydation thermique ou la biométhanisation (tableau 1).

Avant d'entrer dans le détail des techniques, il est utile de rappeler les volumes concernés:

pour une quantité d'huile produite de 17,4 millions de tonnes, I'Europe fait face à plus $d^{\prime} 1,5$ millions de tonnes de co-produits à gérer, tous produits confondus ( $c^{\prime}$ est-à-dire quelque $8 \%$ du produit fini).

\begin{tabular}{|c|c|c|c|c|c|c|}
\hline & $\begin{array}{l}\text { Production } \\
\text { d'huile } \\
\text { Million T }\end{array}$ & $\begin{array}{l}\text { Gommes } \\
10 \exp 3 \mathrm{~T}\end{array}$ & $\begin{array}{c}\text { Pâtes de } \\
\text { neutralisation } \\
\text { 10exp3 T }\end{array}$ & $\begin{array}{c}\text { Terres décolorantes } \\
\text { et charbon actif } \\
\text { 10exp3 } \mathrm{T}\end{array}$ & $\begin{array}{c}\text { Distillats } \\
\text { acide gras } \\
10 \exp 3 \mathrm{~T}\end{array}$ & $\begin{array}{c}\text { Terres } \\
\mathrm{Ni} \\
10 \exp 3 \mathrm{~T}\end{array}$ \\
\hline Colza & 5,5 & 13,8 & 550 & 55 & 33 & 7,7 \\
\hline Soja & 2,6 & 6,5 & 260 & 26 & 15,6 & 3,6 \\
\hline Tournesol & 1,8 & 4,5 & 180 & 18 & 18 & 2,5 \\
\hline Palme & 1,8 & & & 18 & 90 & 2,5 \\
\hline Suif & 1,4 & & & 14 & 8,4 & 2,0 \\
\hline Lard & 1,2 & & & 12 & 7,2 & 1,7 \\
\hline Beurre & 1,2 & & & & & \\
\hline Olive & 0,6 & & 12 & 6 & 64 & 0,8 \\
\hline Autres & 1,3 & 3,2 & 130 & 13 & 7,8 & 1,8 \\
\hline Total & 17,4 & 28 & 1132 & 162 & 244 & 23 \\
\hline
\end{tabular}




\section{Oxydation thermique}

Nous avons étudié le mélange suivant : soit, les co-produits d'un raffinage chimique de $20 \mathrm{~T} / \mathrm{h}$ comprenant: les pâtes de neutralisation, les terres décolorantes et les boues d'un prétraitement physico-chimique des effluents comme on le rencontre en général dans un raffinage chimique. Les boues physico-chimiques sont constituées de $85 \%$ d'eau.

La somme de ces co-produits s'élève à $37 \mathrm{~T} / \mathrm{j}$. Le tout représente un mélange d'une capacité calorifique de 6,4 MW, soit un potentiel de production de vapeur supérieur à $250 \mathrm{~kg}$ de vapeur par tonne d'huile produite (tableau 2). Nous pouvons conclure, sachant que les besoins en vapeur d'une usine s'échelonnent de 200 à $250 \mathrm{~kg}$ de vapeur par tonne d'huile, que l'oxydation thermique des co-produits génère suffisamment d'énergie non seulement pour répondre aux besoins de vapeur process mais aussi, par exemple, pour évaporer des rejets liquides fortement chargés tels les boues physico-chimiques elles-mêmes, les concentrats d'osmose ou les régénérats.

\section{Oxydeur thermique multifluides}

Le schéma de principe de notre oxydeur thermique multifluides, appelé Cinclus ${ }^{\circledR}$, apparaît en figure 1.

Le brûleur est formé d'une succession de tubes concentriques. En principe, chaque tube reçoit un type de combustible. Le brûleur accepte aussi le mélange de cop.

Les cop sont alimentés en tête de combusteur à 1 bar. Ils sont pulvérisés grâce à l'injection d'air d'atomisation à $300 \mathrm{~m} / \mathrm{sec}$. L'air de combustion est injecté en périphérie. Le brûleur est immédiatement solidaire de la chambre d'oxydation : cylindre allongé où règne une température de $950{ }^{\circ} \mathrm{C}$.

La cinétique du jet et la géométrie de chambre engendrent une recirculation intense des gaz : chaque particule fait le tour de la chambre des dizaines de fois en 2 secondes, ce qui assure une combustion optimale.

Le bloc chaudière se place entre la chambre de conditionnement et le filtre. Notre technique consiste à refroidir les émissions sortantes de la chambre d'oxydation de 950 à $700{ }^{\circ} \mathrm{C}$ par un système de recirculation des fumées refroidies à $180^{\circ} \mathrm{C}$ au sortir du filtre à manche. Cette différence de tempérarure suffit à solidifier les particules en fusion avant qu'elles n'atteignent la chaudière au risque de la colmater. En les refroidissant au préalable, elles prennent la forme de cendres qui seront récupérées dans la trémie: une technique qui permet d'éviter l'encrassement de la chaudière.
Tableau 2. Composition de l'échantillon analysé.

\begin{tabular}{|c|c|c|c|c|c|c|}
\hline \multirow[t]{2}{*}{ Raffinage $20 \mathrm{~T} / \mathrm{h}$} & \multicolumn{2}{|c|}{$\begin{array}{c}\text { Quantités } \\
\text { de coproduits }\end{array}$} & \multirow{2}{*}{$\begin{array}{c}\text { PCI } \\
\mathrm{MJ} / \mathbf{k g}\end{array}$} & \multirow{2}{*}{$\begin{array}{c}\text { Puissance } \\
\text { calorifique } \\
\text { MW }\end{array}$} & \multirow{2}{*}{$\begin{array}{c}\text { Capacité } \\
\text { thermique } \\
\text { kW/T.j huile }\end{array}$} & \multirow{2}{*}{$\begin{array}{c}\text { Potentiel } \\
\text { production vapeur } \\
\text { kg/T huile }\end{array}$} \\
\hline & $\mathbf{T} / \mathbf{j}$ & $\%$ & & & & \\
\hline Pâtes & 30 & 80 & 17 & 5,9 & 16 & 236 \\
\hline SBE & 3,6 & 9 & 11 & 0,5 & 1,3 & 18 \\
\hline $\begin{array}{l}\text { Boues physico- } \\
\text { chimiques }\end{array}$ & 4 & 11 & 1 & 0,05 & 0,1 & 2 \\
\hline Total & 37,6 & & & 6,4 & 17,8 & 256 \\
\hline
\end{tabular}

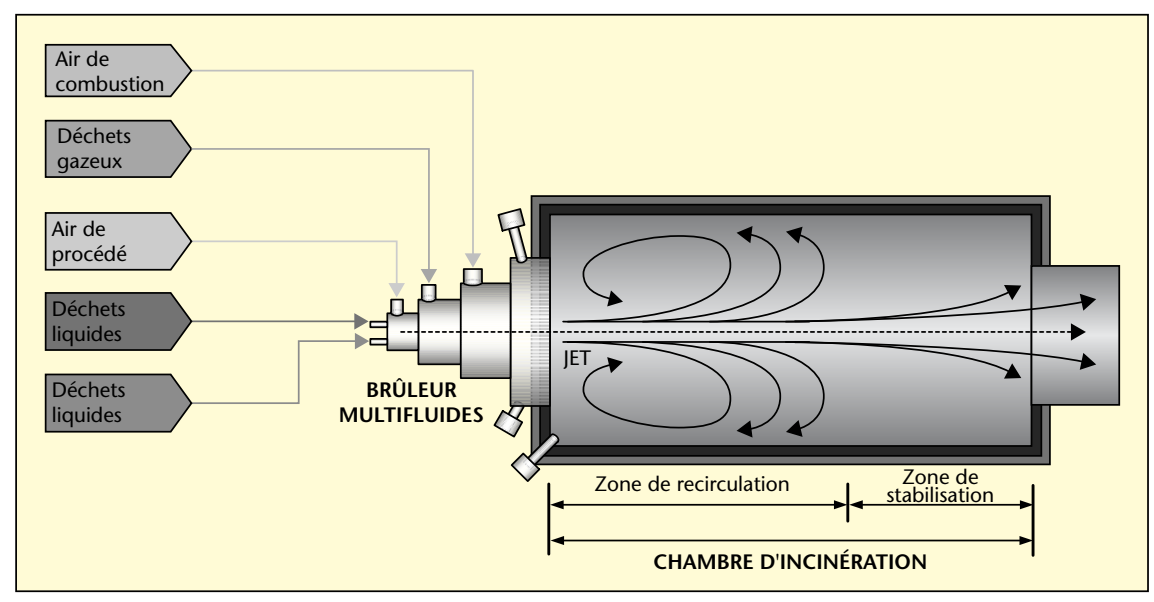

Figure 1. Schéma de principe du combusteur multifluides Cinclus ${ }^{\circledast}$.

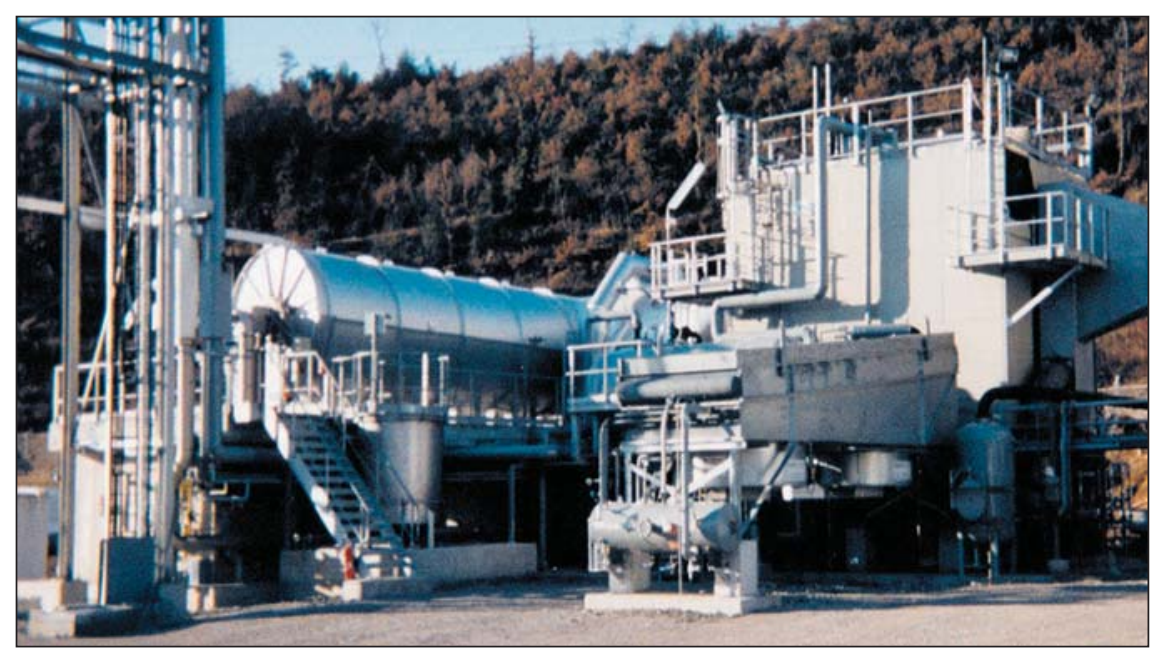

Figure 2. Référence, Sisteron, France. 
En conclusion, retenons que :

- la chambre de combustion est robuste et, grâce à sa conception, évite l'encrassement ;

- le rendement d'oxydation du carbone est total et que les émissions sur notre substrat sont inférieures aux normes ;

- le rendement thermique est supérieur à $70 \%$ et que le rendement électrique est supérieur à $20 \%$.

Enfin, que la solution est flexible et autorise la combustion des différents co-produits, ceux dits « toxiques » également (figure 4).

\section{La digestion anaérobie}

À côté de l'oxydation thermique, nous avons envisagé la digestion anaérobie.

Le procédé est naturel, sans températures ni pressions extrêmes, il met en jeu une biomasse anaérobie thermophile produisant du biogaz utilisable directement à la chaudière. II présente un rendement tout à fait acceptable: $75 \%$ méthane et $40 \%$ de rendement en turbine à gaz.

L'avantage de cette technologie par rapport à I'oxydation thermique est certainement sa grande tolérance (permis).

La digestion anaérobie des cop a été approchée en partenariat avec une société spécialisée et leader en la matière: OWS, Gent, Belgique, dont le réacteur est dénommé Dranco ${ }^{\circledR}$.

Le principe de fonctionnement du réacteur est assez simple : le réacteur est une cuve cylindroconique vide. La matière est alimentée dans la partie sommitale du réacteur, se déplace verticalement au travers du réacteur et est extraite par une vis au niveau du cône.

Le mélange dans le réacteur assuré par recirculation de la matière, pour un volume entrant, 6 volumes sont recirculés.

La digestion anaérobie sur un tel substrat atteint un rendement élevé : $12 \mathrm{~m}^{3}$ biogas $/ \mathrm{m}^{3}$ réacteur.jour.

Le résidu sec (digestat) issu de la digestion constitue certainement $20 \%$ de la quantité initiale. L'avantage de ce sous-produit est qu'il présente une valeur ajoutée non négligeable : après dégazage et compostage partiel, il est transformé en humus de haute qualité (figures 3-4).

Reprenons notre étude de cas où nous avons étudié la digestion anaérobie à partir du mélange de co-produits : le mélange se composait de plus de $50 \%$ de matières sèches, pratiquement $80 \%$ de matières volatiles dont plus de $90 \%$ biodégradables.

Nos espérances en termes de production de biogaz ont été confirmées : $317 \mathrm{~m}^{3}$ biogaz/T. Au niveau du dimensionnement réacteur et du bilan énergétique : I'on retiendra : un réacteur de $2200 \mathrm{~m}^{3}$, une production 3,8 millions de $\mathrm{m}^{3}$ de biogaz par an soit un rendement électri-

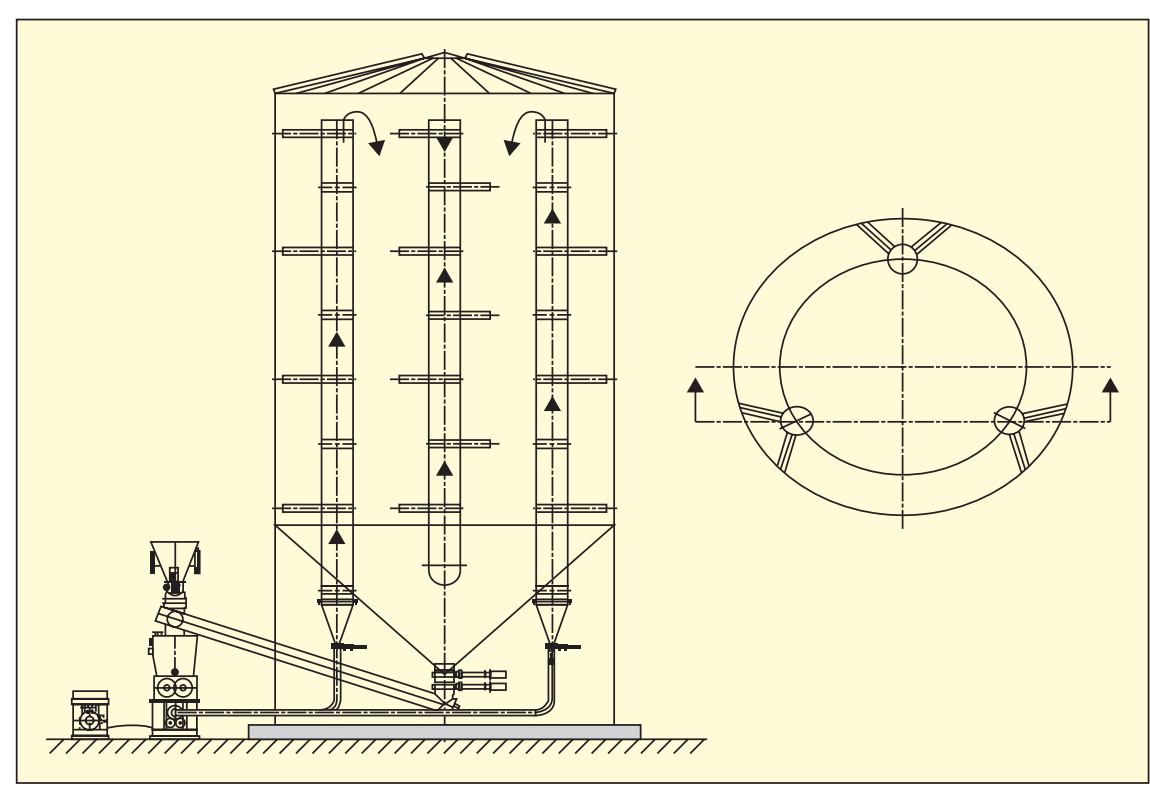

Figure 3. Schéma du digesteur anaérobie.

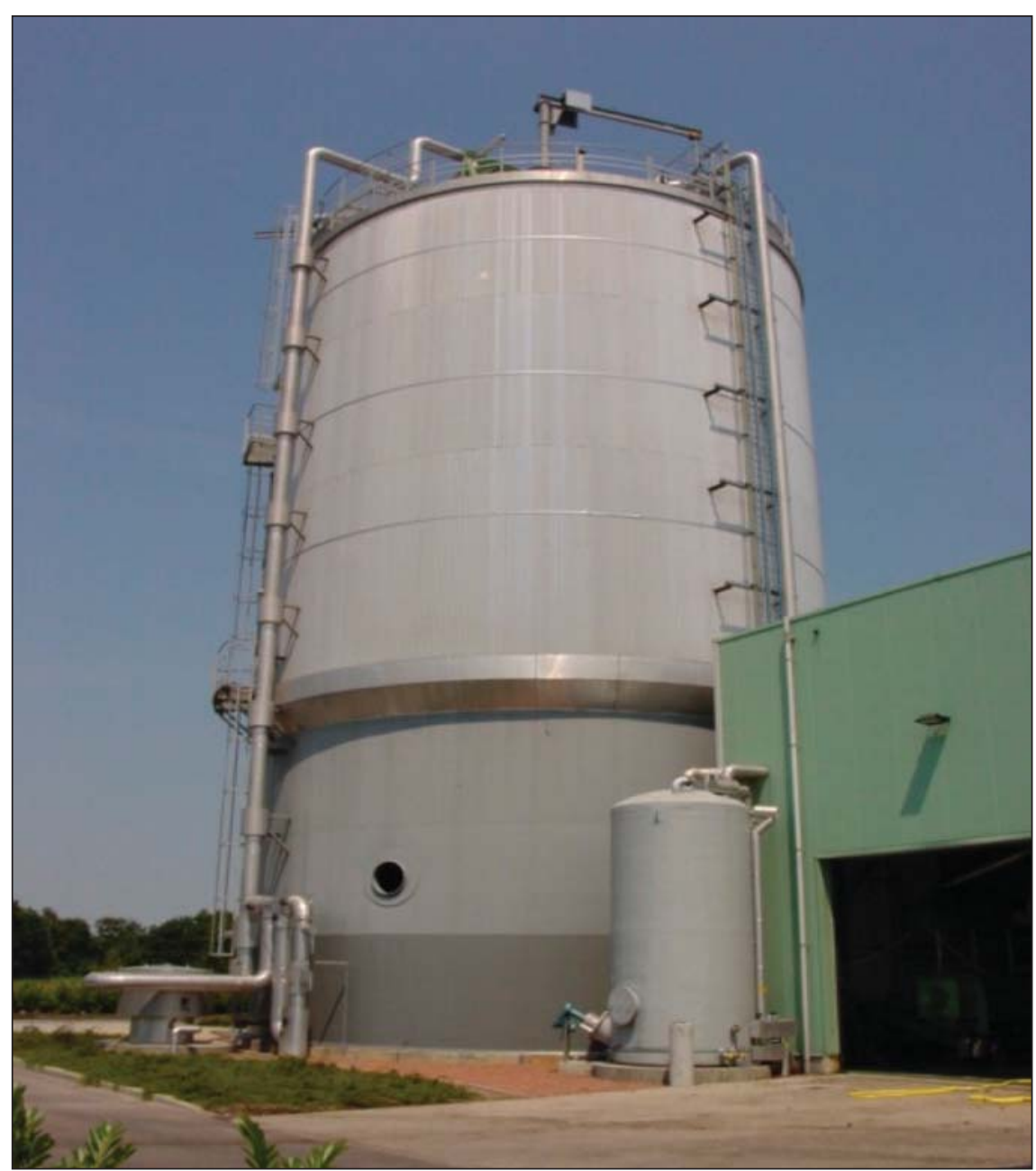

Figure 4. Référence, Brecht, Belgique. 
Tableau 3. Dimensionnement et rendement de la digestion anaérobie.

\begin{tabular}{|lcc|}
\hline Raffinage 20 T/h & T/an & 12000 \\
Co-produits & $\mathrm{m}^{3}$ & 2200 \\
\hline Volume digesteur : & $\mathrm{m}$ & 12,5 \\
Diamètre : & $\mathrm{m}$ & 21,4 \\
Hauteur : & 10 exp $6 \mathrm{~m}^{3} / \mathrm{an}$ & 3,8 \\
\hline Biogas en million $\mathrm{m}^{3}$ & & 1,3 \\
30 GWh/an, 3,5MW & $\mathrm{MW}$ & \\
\hline Électricité Puissance & & 10,3 \\
Nominale & $\mathrm{GWh} / \mathrm{an}$ & \\
\hline Production totale & & \\
\hline
\end{tabular}

Tableau 4. Bilan économique.

\begin{tabular}{|lcc|}
\hline $\begin{array}{l}\text { Raffinage 20 T/h } \\
\text { Co-produits }\end{array}$ & T/an & 12000 \\
\hline Investissement & 10exp6EUR & 3 \\
\hline Coût/Tonne & & \\
\hline - Amortissement (15 ans, 7 \%) & EUR/T & 23,1 \\
O \& M & EUR/T & 14,5 \\
\cline { 2 - 3 } Total & EUR/T & 37,6 \\
\hline Revenus/Tonne & & \\
Électricité $(10$ \&/kWh) & EUR/T & 85 \\
\hline Revenu net & EUR/T & 47,4 \\
\hline
\end{tabular}

que de 1,3 MW, ce qui correspond à une production électrique de $10 \mathrm{GWh} / \mathrm{an}$ (tableau 3).

En résumé, le traitement des cop, dont celui des pâtes de neutralisation, apporterait les avantages suivants :

- absence d'eaux acides (problème des SO4), - absence d'interphase,

- absence de consommation en réactifs chimiques (acide sulfurique),

- diminution du combustible fossile (production d'énergie).

Le bilan économique est certainement positif (tableau 4).

La plus-value des huiles acides est à comparer au bilan de digestion anaérobie :

Valeur nette 2,5 $₫ / \mathrm{kg}: 25$ eur/ T pâtes; Valorisation énergétique : 47 eur/ T pâtes ; Certificat Vert : 71 eur/ T pâtes.

\section{Conclusion}

L'application d'une telle technologie in situ permettrait à la fois de résoudre des problèmes d'élimination de résidus, de suppression de certains effluents problématiques et d'atteindre un bilan énergétique dans l'usine pratiquement en autosuffisance. En 2020, en Europe, 2 millions de tonnes de co-produits devront être gérés. Parmi toutes les applications envisageables la production d'énergie devrait être l'orientation la plus appropriée. 